\title{
Gamma-band modulation in the human amygdala during reaching movements
}

\author{
Angad S. Gogia, BS, ${ }^{1}$ Roberto Martin Del Campo-Vera, PhD, ${ }^{1}$ Kuang-Hsuan Chen, $\mathrm{PhD},{ }^{1}$ \\ Rinu Sebastian, MS, ${ }^{1}$ George Nune, MD, ${ }^{2,3}$ Daniel R. Kramer, MD, ${ }^{1,3}$ Morgan B. Lee, BS, ${ }^{1}$ \\ Ali R. Tafreshi, MD, ${ }^{1}$ Michael F. Barbaro, BA, ${ }^{1}$ Charles Y. Liu, MD, PhD, ${ }^{1,3,4}$ Spencer Kellis, PhD, ${ }^{1,3-5}$ \\ and Brian Lee, MD, PhD $1,3,4$
}

Departments of ${ }^{1}$ Neurological Surgery and ${ }^{2}$ Neurology and ${ }^{3}$ USC Neurorestoration Center, Keck School of Medicine of USC, Los Angeles; and ${ }^{4}$ Department of Biology and Biological Engineering and ${ }^{5}$ Tianqiao and Chrissy Chen Brain-Machine Interface Center, Chen Institute for Neuroscience, California Institute of Technology, Pasadena, California

OBJECTIVE Motor brain-computer interface $(\mathrm{BCl})$ represents a new frontier in neurological surgery that could provide significant benefits for patients living with motor deficits. Both the primary motor cortex and posterior parietal cortex have successfully been used as a neural source for human motor $\mathrm{BCl}$, leading to interest in exploring other brain areas involved in motor control. The amygdala is one area that has been shown to have functional connectivity to the motor system; however, its role in movement execution is not well studied. Gamma oscillations $(30-200 \mathrm{~Hz})$ are known to be prokinetic in the human cortex, but their role is poorly understood in subcortical structures. Here, the authors use direct electrophysiological recordings and the classic "center-out" direct-reach experiment to study amygdaloid gamma-band modulation in 8 patients with medically refractory epilepsy.

METHODS The study population consisted of 8 epilepsy patients (2 men; age range 21-62 years) who underwent implantation of micro-macro depth electrodes for seizure localization and EEG monitoring. Data from the macro contacts sampled at $2000 \mathrm{~Hz}$ were used for analysis. The classic center-out direct-reach experiment was used, which consists of an intertrial interval phase, a fixation phase, and a response phase. The authors assessed the statistical significance of neural modulation by inspecting for nonoverlapping areas in the $95 \%$ confidence intervals of spectral power for the response and fixation phases.

RESULTS In 5 of the 8 patients, power spectral analysis showed a statistically significant increase in power within regions of the gamma band during the response phase compared with the fixation phase. In these 5 patients, the $95 \%$ bootstrapped confidence intervals of trial-averaged power in contiguous frequencies of the gamma band during the response phase were above, and did not overlap with, the confidence intervals of trial-averaged power during the fixation phase.

CONCLUSIONS To the authors' knowledge, this is the first time that direct neural recordings have been used to show gamma-band modulation in the human amygdala during the execution of voluntary movement. This work indicates that gamma-band modulation in the amygdala could be a contributing source of neural signals for use in a motor $\mathrm{BCl}$ system. https://thejns.org/doi/abs/10.3171/2020.4.FOCUS20179

KEYWORDS gamma band; amygdala; direct reach; stereo-EEG; human; electrophysiology

$\mathrm{R}$ ECENT advances in neuroscience and technology are creating novel opportunities to use implantable devices to repair the nervous system in ways that were not previously possible. ${ }^{1-3}$ Specifically in neurosurgery, implantable devices, including deep brain stimulators, vagus nerve stimulators, and spinal cord stimulators, are used to treat a variety of conditions such as Parkinson's disease, epilepsy, and chronic pain. ${ }^{4-6}$ These devices, along with advances in biomedical engineering, represent a new frontier for the field of brain-computer interface (BCI). Motor $\mathrm{BCI}$ is one specific example of BCI focused on restoring motor function and could provide significant benefits for persons living with motor deficits from conditions such as stroke, amyotrophic lateral sclerosis, or spinal cord injury. ${ }^{7-9}$ Early work with motor BCI focused on decoding signals directly from the primary motor cortex, given its

ABBREVIATIONS BCI = brain-computer interface; ITI = intertrial interval; PPC = posterior parietal cortex.

SUBMITTED March 1, 2020. ACCEPTED April 14, 2020.

INCLUDE WHEN CITING DOI: 10.3171/2020.4.FOCUS20179. 
known function in controlling movement. Indeed, neurons in the primary motor cortex were shown to have firing patterns associated with a variety of movement parameters, including velocity, acceleration, and force..$^{10-13}$ These signals have been successfully utilized to control both physical and virtual effectors. ${ }^{12,14-16}$ There has also been interest in recording signals from higher-order brain areas. One such area is the posterior parietal cortex (PPC), a brain region known to be involved in generating motor plans that are then executed by the primary motor cortex..$^{17-19}$ The PPC processes cognitive-level aspects of sensorimotor control, including sensory inputs, goals and trajectories of movement, and both ipsilateral and contralateral limb movements. ${ }^{20-24}$ The PPC has been used successfully as a neural source in human motor BCI. ${ }^{16,17,25}$

Given the success of utilizing higher-order brain areas beyond the motor cortex in motor BCI, it is possible that other brain areas involved in the motor system may also provide suitable targets for recording and decoding aspects of movement. One such area is the amygdala, consisting of a complex set of medial temporal lobe nuclei, best known for its role in processing fearful stimuli. ${ }^{26-28}$ However, there is emerging evidence that the amygdala may also modulate the motor system in humans..$^{29}$ It is structurally connected to the motor cortex through the external capsule ${ }^{30}$ and functionally to other parts of the motor system, including the subthalamic nucleus ${ }^{31}$ and globus pallidus. ${ }^{32} \mathrm{MRI}$ analysis of resting-state connectivity between the amygdala and the striatum suggests that it is also associated with freezing of gait in patients with Parkinson's disease, ${ }^{33}$ providing further evidence that the amygdala may be involved in motor control. However, its exact role in movement is not clear.

A major benefit of utilizing direct intracranial neural recordings is that they provide high temporal and spatial resolution compared with other noninvasive methods such as functional MRI. ${ }^{34,35}$ Developments in electrode technology and signal analysis techniques have increased our understanding of human behavior, ${ }^{36}$ as they allow us to analyze these signals during various cognitive and motor tasks. Local field potentials, the linear sum of overlapping electrical fields created by sources of current across the membrane of a cell, ${ }^{37}$ have been used to show that neuronal oscillations in the various frequency bands have functional significance. ${ }^{38-40}$ Using stereo-EEG, these recordings are often performed in epileptic patients who are undergoing presurgical evaluation. ${ }^{41}$

In humans, 40- to $80-\mathrm{Hz}$ oscillations have been observed in the amygdala in response to aversive visual stimuli, ${ }^{42}$ and lower frequencies between 15 and $35 \mathrm{~Hz}$ have been shown to be involved in amygdaloid olfactory processing. ${ }^{43}$ Single-neuron studies have also demonstrated specificity for facial features in both human and nonhuman primate amygdalae. ${ }^{44}$ In rodents, optogenetic induction of 4-Hz oscillations in the prefrontal cortex activates the amygdala and elicits fear behavior. ${ }^{45}$ There is also evidence of gamma-band (defined as $>30 \mathrm{~Hz}$ in that particular study) power changes in the rodent amygdala in response to human faces ${ }^{46}$ Older rats have increased betaband $(13-30 \mathrm{~Hz})$ power in the amygdala compared with younger rats during decision-making tasks. ${ }^{47}$
In this study, we focus on the entire range of frequencies between 30 and $200 \mathrm{~Hz}$. This range includes both what are typically known as the gamma $(30-100 \mathrm{~Hz})$ and high gamma (100-200 Hz) frequency bands. ${ }^{48,49}$ However, there is no consensus on the cutoffs that separate the two frequency bands. Studying the entire range from 30 to $200 \mathrm{~Hz}$ is an approach that has previously been taken to encompass the spectrum of heterogeneous gamma-band definitions in the neurophysiology literature..$^{50}$ Here, we use the term "gamma band" to discuss the entire range of frequencies between 30 and $200 \mathrm{~Hz}$. Frequency oscillations between this entire range are related to neuronal firing rates ${ }^{51-53}$ and may be the result of either oscillatory field potentials or nonoscillatory, asynchronous changes in mean population activity. ${ }^{54}$ Furthermore, previous studies have implicated power modulation between 30 and $200 \mathrm{~Hz}$ in motor control in both humans and nonhuman primates. ${ }^{53,55-59}$ In the cortical brain, gamma oscillations have been shown to be associated with movement. ${ }^{57}$ However, the role of gamma oscillations in subcortical structures is complex and not well characterized. ${ }^{60}$ Here, we used direct electrophysiological recordings and the classic "center-out" direct-reach paradigm to study amygdaloid gamma-band modulation in 8 patients with medically refractory epilepsy.

\section{Methods \\ Patients}

This study includes 8 epilepsy patients $(2$ men; age range 21-62 years), all with micro-macro depth leads implanted for epilepsy monitoring and seizure localization. Lead configuration and placement were individualized for each patient after extensive workup (video-EEG monitoring, MRI, and PET imaging) and discussion at an interdisciplinary conference that included epileptologists, neurosurgeons, and neuroradiologists. Five patients had bilateral amygdaloid placement, while 3 had implants in the right amygdala only. Patient characteristics, including number and location of leads in the amygdala, are presented in Table 1. The location of implanted leads was confirmed by merging the postoperative CT scans with the planned trajectories on preoperative MRI scans. Figure 1 shows coronal, axial, and sagittal views of a representative electrode implanted in the right amygdala of an example patient. Each patient gave written, informed consent to participate in this study, which was approved by the institutional review board of the University of Southern California, Health Sciences Campus.

\section{Neural Signal Acquisition}

All participants had micro-macro depth leads implanted (MM16A-SP05x-000, Ad-Tech Medical Instrumentation Corp.) in the amygdala. Leads were cylindrical in shape (diameter $=1.3 \mathrm{~mm}$, length $=370 \mathrm{~mm}$ ) with six $1.57-\mathrm{mm}$ long platinum contacts spaced $5 \mathrm{~mm}$ apart from center to center, and 10 micro contacts interspersed between the macro contacts made of 50.8- $\mu \mathrm{m}$-diameter platinum wire. The neural signals used in this study were acquired from only the macro-type electrode contacts located in gray matter (NeuroPort System, Blackrock Microsystems) us- 
TABLE 1. Patient profiles

\begin{tabular}{|c|c|c|c|c|c|}
\hline $\begin{array}{l}\text { Patient } \\
\text { No. }\end{array}$ & $\begin{array}{l}\text { Age (yrs), } \\
\text { Sex }\end{array}$ & Handedness & Seizure Onset Zone & $\begin{array}{l}\text { Implantation } \\
\text { Side }\end{array}$ & No. of Leads \\
\hline P024 & $45, \mathrm{~F}$ & Rt & Rt insula \& frontal operculum & Bilateral & Rt: 1 ; It: 1 \\
\hline P025 & $40, F$ & Rt & $\begin{array}{l}\text { Lt hippocampus, rt hippocampus/amyg- } \\
\text { dala, \& rt frontal }\end{array}$ & Bilateral & Rt: 1 ; It: 1 \\
\hline P026 & $37, \mathrm{M}$ & NA & NA & Bilateral & Rt: 1 ; It: 1 \\
\hline P027 & $46, F$ & $\mathrm{Rt}$ & Rt orbitofrontal & Bilateral & Rt: 1 ; It: 1 \\
\hline P029 & $21, \mathrm{~F}$ & Rt & NA & Bilateral & Rt: $1 ;$ It: 1 \\
\hline P032 & $62, \mathrm{M}$ & $\mathrm{Rt}$ & Rt orbitofrontal & Rt & Rt: 1 \\
\hline P033 & $26, F$ & $\mathrm{Rt}$ & Rt hippocampus & Rt & Rt: 1 \\
\hline P034 & $21, \mathrm{~F}$ & Rt & Rt hippocampus & $\mathrm{Rt}$ & Rt: 1 \\
\hline
\end{tabular}

$\mathrm{NA}=$ not available.

ing a reference contact located in white matter with lowvoltage amplitude as identified by a clinical epileptologist. Re-referencing of the neural signal was not performed. The Blackrock neural acquisition system was configured to record neural signals to disk at 30,000 samples/sec and 2000 samples/sec; the 2000 -samples/sec recording was used for this analysis. The ground of the Blackrock signalprocessing unit was connected to the ground contact of the breakout box of the epilepsy monitoring unit (Natus Quantum LTM Amplifier, Natus Medical Inc.).

\section{Center-Out Direct-Reach Task}

The movement task used in this study was programmed in MATLAB (2018b, The MathWorks Inc.) using the Psychophysics Toolbox Version 3 (PTB-3). ${ }^{61-63}$ It was presented to the study participants on a touch-screen monitor (21.5-inch LED-backlit, $1920 \times 1080$ pixels, $250 \mathrm{~cd} /$ $\mathrm{m}^{2}$ luminance, S2240TB, Dell Inc.). The task comprised 3 separate phases (Fig. 2). After an intertrial interval (ITI) lasting 1-2 seconds, the patient was instructed to raise their right hand and point to a gray circle $(9.53-\mathrm{mm}$ radius) in the center of the screen during the fixation phase (1-4 seconds). Then, in the response phase, a white circle (15.88-mm radius) appeared in pseudo-random order in one of 8 target locations equidistant from the fixation circle (114.3 mm center to center; Fig. 2C). The participant

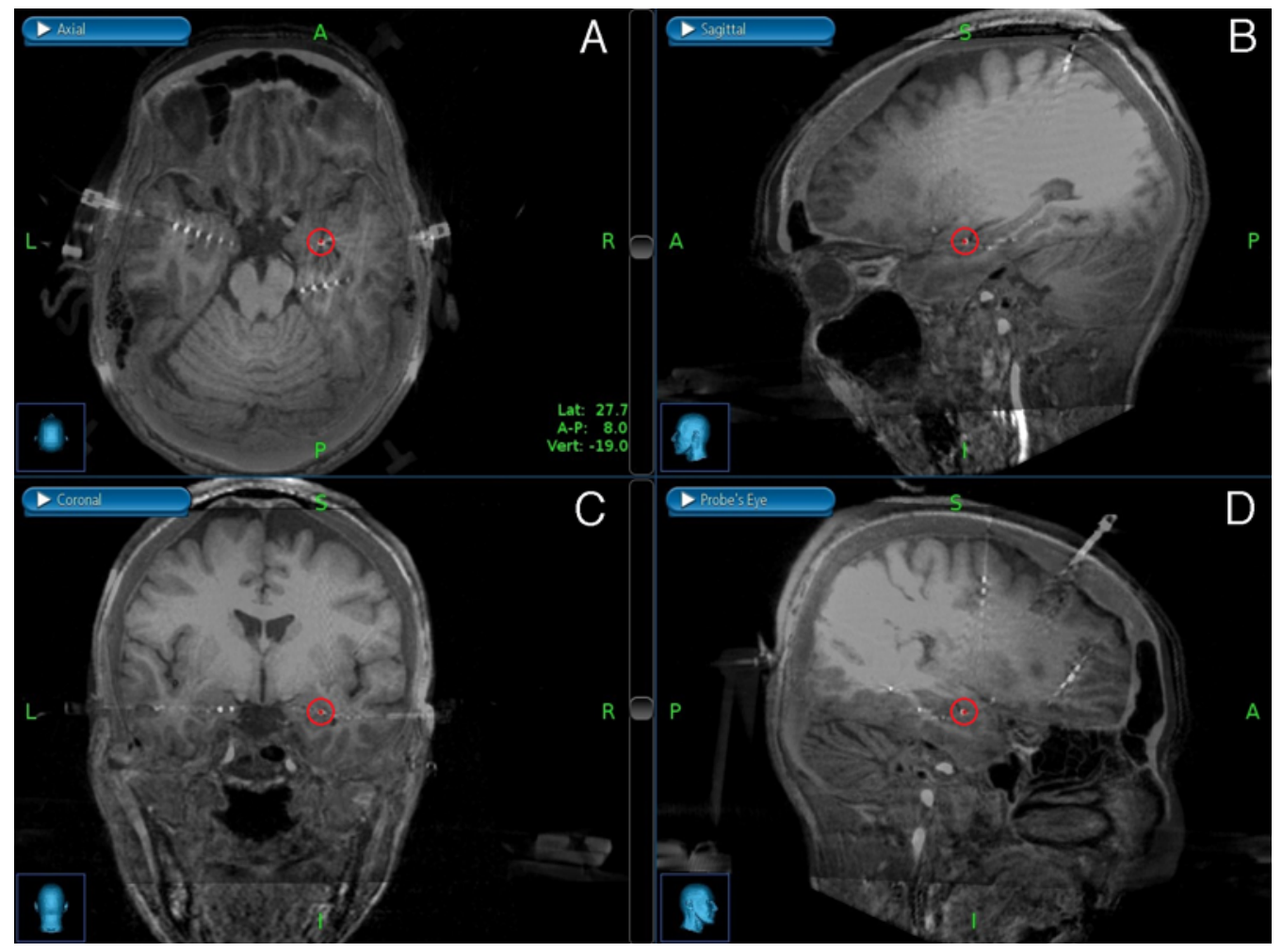

FIG. 1. Merged axial (A), sagittal (B), coronal (C), and probe's-eye (D) CT/MRI images indicating lead placement in the right amygdala and a sample electrode contact (circled in red) from which the results in Fig. 3 (P024) and Fig. 4 were obtained. 


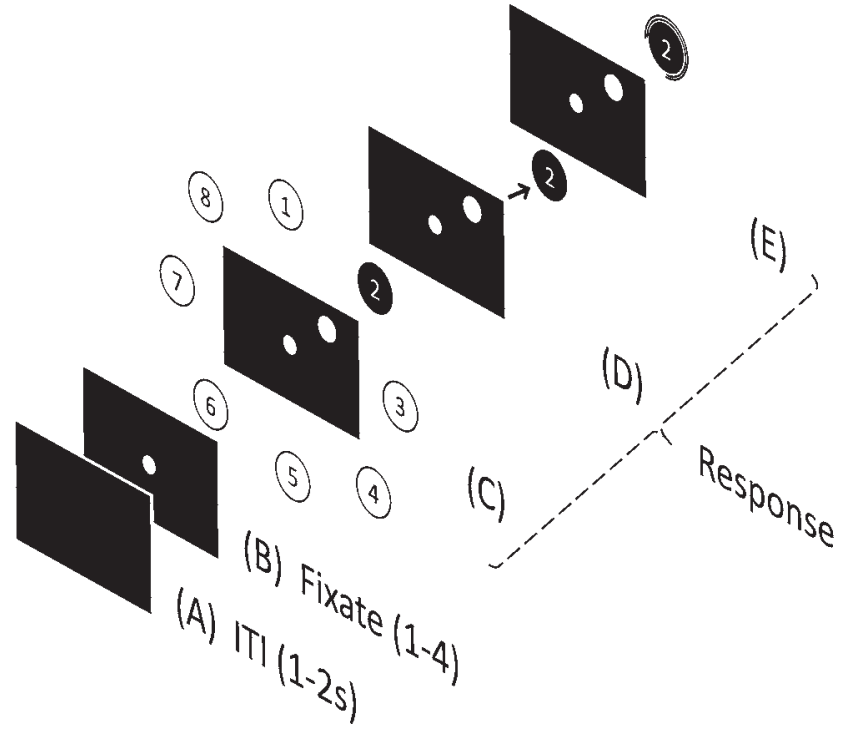

FIG. 2. The phases of the center-out direct-reach task. A: The ITI phase (1-2 seconds), during which the screen is blank. B: The fixation phase (1-4 seconds), during which the patient raises their right hand and points to the gray dot in the center of the screen. C-E: The response phase, during which a target appears at one of 8 equidistant, pseudorandom locations $(C)$; the patient must move toward (D) and double-tap on the target $(\mathrm{E})$.

was instructed to reach with their right arm to double-tap the screen at the location of the target as soon as it appeared. Each run of the task consisted of 64 trials ( 8 per target location). Patients were instructed to fix their gaze to the center of the screen throughout the task and to use peripheral vision to spot the targets.

\section{Neural Signal Processing}

Trials with unsuccessful task behavior, including a double-tap at an incorrect location or response time less than $500 \mathrm{msec}$ (indicating an accidental response), were excluded from analysis. Additionally, trials for which the neural data exhibited spectral power greater than 1.5 times the interquartile range (computed across trials) were excluded from analysis. Finally, trials containing interictal spikes (identified by manual inspection) were also excluded from analysis. Multitaper power spectrums of the remaining trials were performed using Chronux ${ }^{64}$ in MATLAB with 9 leading tapers, a time-bandwidth product of 5, and each phase zero-padded to 2048 samples. Spectrograms were computed using the same parameters as the power spectrums with the addition of the definition of a moving window of 0.5 seconds and step size of 0.05 seconds.

\section{Individual Analysis}

For each phase of the task (ITI, fixation, and response), trial-averaged spectral power density was calculated for each patient. Due to variable response times, 0.05-0.72 seconds was trimmed from the beginning and end of each phase to focus the analysis on the neural response specific to that phase. Confidence intervals were bootstrapped for each frequency bin $(\mathrm{n}=10,000, \alpha=0.05){ }^{65}$ Areas of $95 \%$ confidence interval nonoverlap between the response-phase and fixation-phase spectral power density were identified for additional statistical hypothesis testing. Within those regions, a nonparametric paired-sample test (Wilcoxon signed-rank test) for averaged spectral power was conducted.

\section{Group-Level Analysis}

Trials were combined and averaged across the patients with significant power change during the response phase (compared with the fixation phase) in order to relate the intersubject variability. Each individual's significant frequency values were normalized to the range with significant individual response change, and power spectral values were normalized between the maximum and minimum power values within that frequency range. This normalization was performed by a linear transformation of the form: $f(x)=a x+b$, where $a=1 /\left(x_{\text {max }}-x_{\text {min }}\right), b=-x_{\text {min }}$ l $\left(x_{\max }-x_{\text {min }}\right)$. This generates frequency and power spectral values between zero and one and is also known as data scaling.

The group-level analysis was split into frequency bins of $10 \%$ width. The first bin between $[0,0.1)$ consisted of the statistically significant response-phase power spectral values from the lowest $10 \%$ frequency range of the across-patient combined trials. Power spectral values falling within the $10 \%-20 \%$ frequency range were grouped in the bin between $[0.1,0.2)$ and so on for the remaining bins up to the 10 th bin between $[0.9,1]$. For each frequency bin, the mean and bootstrapped $95 \%$ confidence intervals $(n=$ 10,000) were determined using the combined 306 trials among patients from Table 2 .

\section{Results}

In 5 of 8 patients, trial-averaged power spectral analysis revealed a statistically significant increase in power within contiguous regions of the gamma band during the response phase of the movement trial compared with the fixation-phase baseline. The $95 \%$ confidence intervals of trial-averaged power in the response phase were greater than and did not overlap with the confidence intervals of the fixation phase (bootstrapped, $n=10,000, \alpha$ $=0.05$ ) within the regions shown in Fig. 3. Nonoverlapping regions between confidence intervals were used to determine contiguous frequency ranges within the gamma band. Frequency ranges were calculated by localizing the intersection points between the lower confidence level of the response trial-averaged power and the upper confidence level of the fixation trial-averaged power. For each of these 5 patients, the significant frequency ranges, trialaveraged power in the response and fixation phases, and the percentage power change during movement are presented in Table 2.

Figure 3A shows trial-averaged power spectral densities for the averaged gamma power in nonoverlapping regions during voluntary movement for each patient. Figure $3 \mathrm{~B}$ shows boxplot results indicating the frequency range within gamma-band power that was significantly increased in the response phase compared with the fixation phase. In the 5 patients, the average power increased $21.7 \%-48.3 \%$ between the response and fixation phases in 
TABLE 2. Individual and averaged response across patients with significant gamma-band power increase

\begin{tabular}{|c|c|c|c|c|c|}
\hline Patient No. & $\begin{array}{l}\text { Frequency Range, } \\
\qquad \mathrm{Hz}^{*}\end{array}$ & $\begin{array}{c}\text { Fixation Averaged } \\
\text { Power, } \mu \mathrm{V}^{2} / \mathrm{Hz} \dagger\end{array}$ & $\begin{array}{c}\text { Response } \\
\text { Averaged Power, } \\
\mu \mathrm{V}^{2} / \mathrm{Hz} \dagger\end{array}$ & $\begin{array}{l}\% \text { Change } \\
\text { of Averaged } \\
\text { Powerf }\end{array}$ & $\begin{array}{l}\text { No. of } \\
\text { Successful } \\
\text { Trials }\end{array}$ \\
\hline P024 & {$[73.0,107.0]$} & $0.0605(0.0124)$ & $0.0865(0.0198)$ & $42.98 \% * * *$ & 63 \\
\hline P025 & {$[118.2,128.9]$} & $0.0246(0.0072)$ & $0.0338(0.0100)$ & $37.40 \% * * *$ & 54 \\
\hline P026 & {$[139.6,155.3]$} & $0.0236(0.0060)$ & $0.0350(0.0104)$ & $48.31 \%^{* * *}$ & 61 \\
\hline P027 & {$[30.0,40.0]$} & $0.5451(0.1779)$ & $0.6634(0.2389)$ & $21.70 \% * *$ & 64 \\
\hline P029 & {$[136.7,156.3]$} & $0.0120(0.0020)$ & $0.0160(0.0035)$ & $33.33 \% * * *$ & 64 \\
\hline Average & {$[0,1]$} & $0.3962(0.2732)$ & $0.4850(0.2853)$ & $22.41 \%$ *** & 306 total \\
\hline
\end{tabular}

the nonoverlapping confidence interval regions (Wilcoxon signed-rank test, $\mathrm{p}<0.01$ in patient P027 and $\mathrm{p}<0.001$ in all others). Merged CT/MRI images that indicate lead placement in the amygdala with coronal, sagittal, axial, and probe's-eye views are shown in Fig. 3C. The corresponding gray matter electrode contact-from which the results in the first two columns were derived-is highlighted with a red circle.

To characterize the magnitude of the gamma-band change across patients with significant modulation, their frequency range was normalized between 0 and 1 for each patient as described in Methods. Across their combined 306 successful trials, the average power magnitude change was $22.41 \%$ (Wilcoxon signed-rank test, $\mathrm{p}<0.001$ ). These results are presented in the last row of Table 2. Additionally, Fig. 3D and E displays the combined regions' significant differences in the gamma band during the response phase across patients.

In these 5 patients, the power increase with the contiguous frequency range was limited to the response phase of the trial and with respect to the fixation phase. A return to baseline level in the gamma band was observed during the ITI phase of the subsequent trial. This comparison was done using the nonoverlapping region of the $95 \%$ confidence intervals of the trial's response phase when compared with the subsequent ITI phase. A representative patient's (P024) trial-averaged power spectral density including the $95 \%$ confidence intervals for each trial phase is presented in Fig. 4 upper. This increase in power with the subsequent rebound can be appreciated in Fig. 4 lower, which shows a spectrogram for a representative patient trial.

Although the gamma band showed the most modulation in the highest number of patients, there were other statistically significant changes in various frequency bands seen during the response phase of the trial compared with the fixation phase. Three patients showed a decrease in beta-band power for intervals for contiguous regions of the beta band $(13-30 \mathrm{~Hz}), 1$ showed an increase in the theta band $(4-8 \mathrm{~Hz})$, and 1 showed a decrease in the alpha band $(8-13 \mathrm{~Hz})$ (bootstrapped, $\mathrm{n}=10,000, \alpha=0.05)$.

\section{Discussion}

To our knowledge, this is the first time that direct neural recordings have been used to show gamma-band modulation in the human amygdala during the execution of voluntary movement. In this study, we showed that 5 of 8 patients had a statistically significant increase in power in contiguous regions of nonoverlap in the $95 \%$ confidence intervals between the fixation and response phases during the center-out direct-reach task. Because the patient does not move during the fixation phase, it is likely that the modulation we observe is specific for movement execution. In addition, the randomly ordered display of target locations to one of 2 possible places was used to mitigate the contribution of motor planning signals to our findings. Our findings suggest that the human amygdala plays a role in movement execution.

Notably, while there is literature linking amygdala activity to motor system inhibition as previously described, until now there has been limited work showing how these amygdala-motor connections may be involved in increasing motor output or motor execution. In one study, Voon et al. showed an increase in right amygdala activity using functional MRI in patients with positive motor symptom conversion disorder compared with healthy controls. ${ }^{66}$ Although that study only had 11 patients and was limited to those with conversion disorder, it provided the first evidence that amygdala activity may be associated with increased motor output. Our findings here build on that work by exploring voluntary movement in patients utilizing direct intracranial neural recordings.

Our findings indicate that amygdala gamma-band modulation may be a suitable neural signal for use in a motor BCI system. In 2013, Engelhard et al. reported using operant conditioning in nonhuman primates to induce low gamma (30-50 Hz) power changes in the motor cortex for accurate use in a brain-machine interface as recorded by a 96-microelectrode array. ${ }^{58}$ Our findings indicate that it may be feasible to do the same in humans with signals from the amygdala. In that paper, the authors specifically looked at low gamma frequencies because of their known relation- 

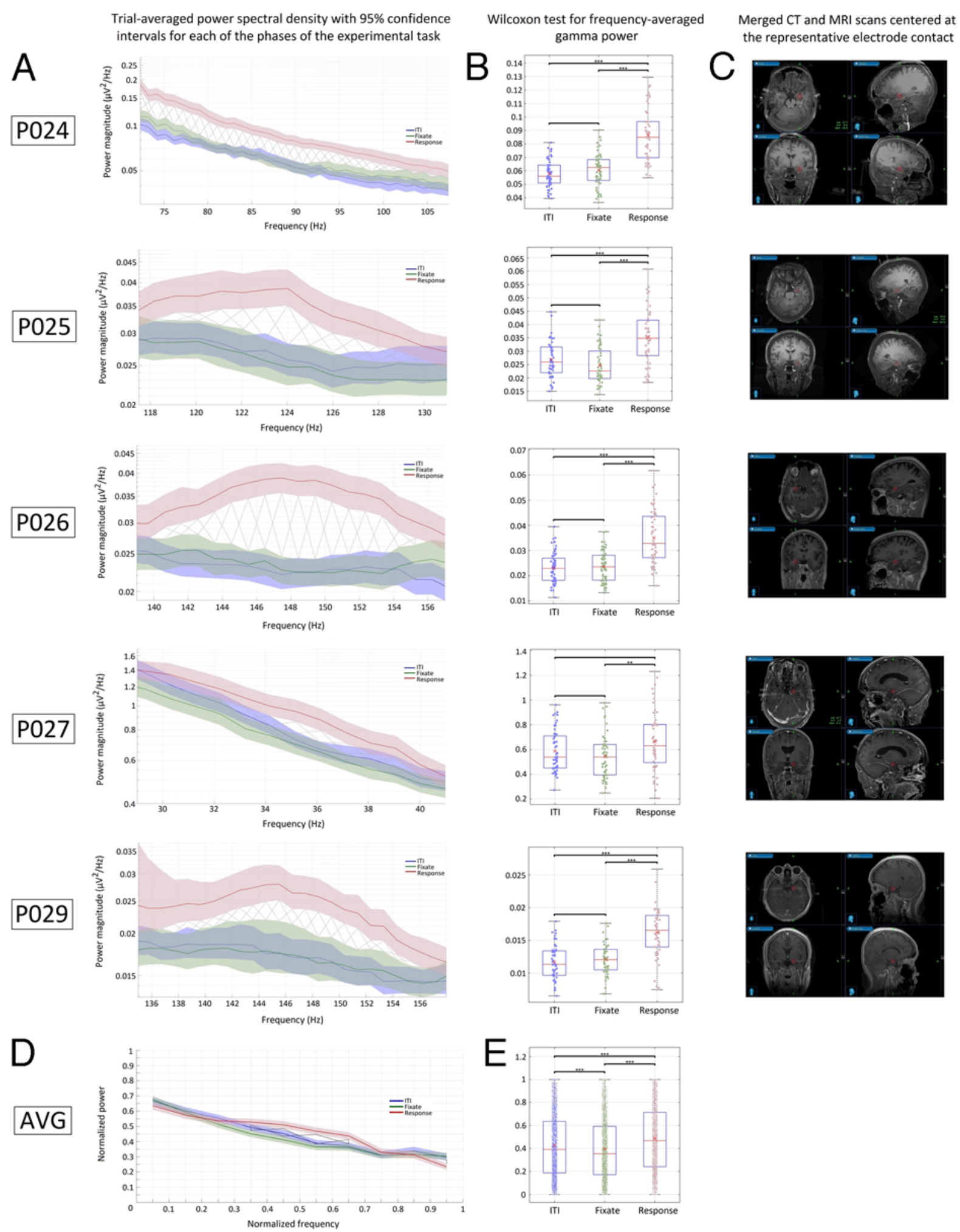

FIG. 3. Power spectral densities, averaged gamma power, and merged CT/MR images for each patient with significant gamma-band modulation during reaching movement. A: Trial-averaged spectral power density for the 3 experimental task phases for each patient. Color-shaded areas represent $95 \%$ confidence intervals for the various experimental phases. B: Boxplots showing trial-averaged gamma-band power in the amygdala for the significant patient contact. Gamma-band power was significantly increased in the response phase compared with the fixation phase (Wilcoxon signed-rank test: ** $<0.01 ;{ }^{* * *} p<0.001$ ). C: Merged axial (upper left), sagittal (upper right), coronal (lower left), and probe's-eye (lower right) CT/MR images indicating lead placement. The circled contact is the same as the contact used in the boxplot and the trial-averaged spectrograms. D and E: Power spectral densities (D) and boxplots $(E)$ for information averaged across all patient trials and across all patients. The frequency range of significant modulation was scaled between 0 and 1 for each patient to allow combination across patients given the variable significant frequency ranges between patients. 

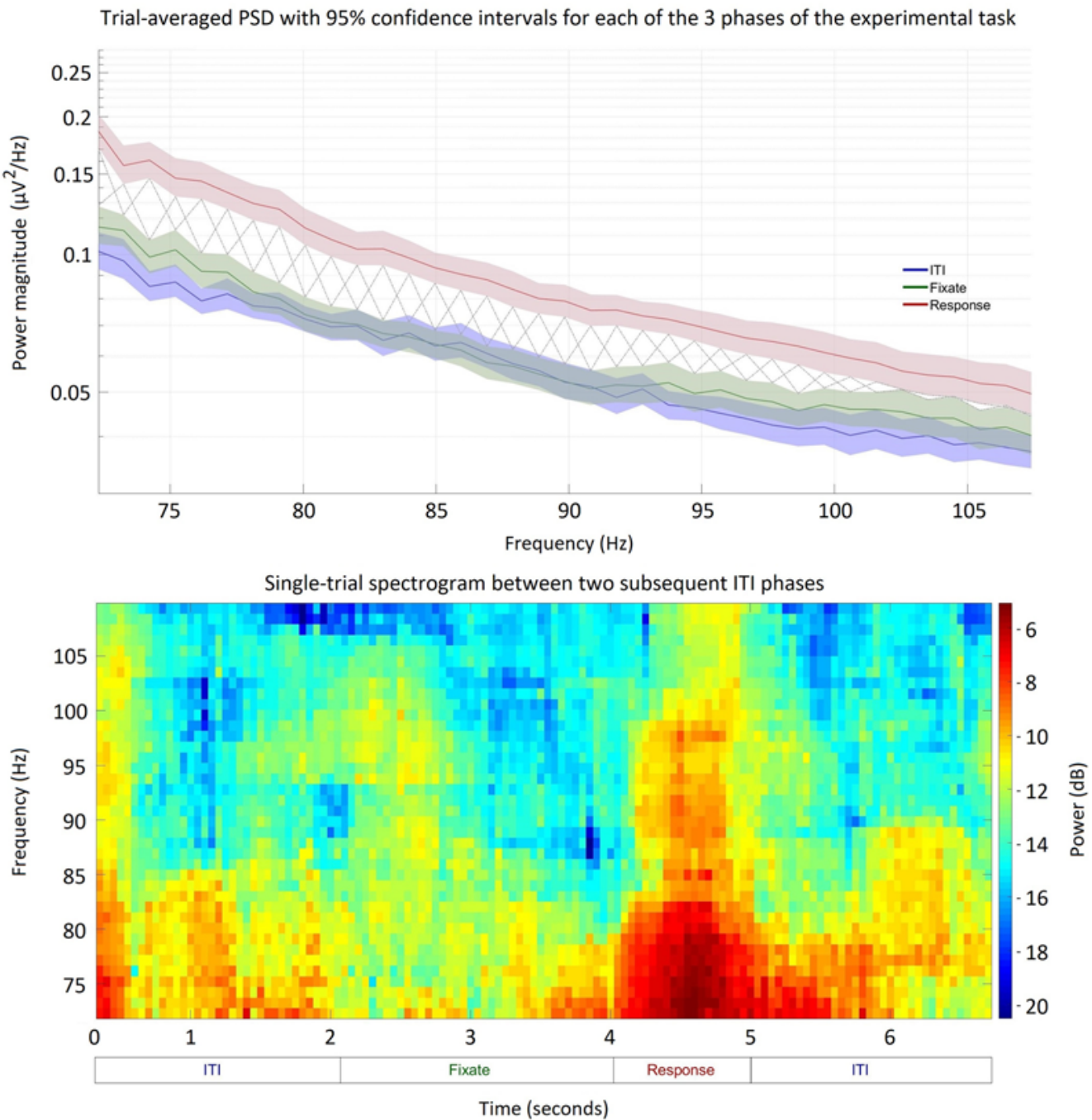

FIG. 4. Upper: Representative trial-averaged power spectral density (PSD) for the 3 phases of the experimental task for patient P024. The color-shaded areas represent the $95 \%$ confidence intervals. The rhomboid pattern indicates nonoverlapping areas of the $95 \%$ confidence intervals between the response and fixation phases. Lower: Representative ITI-to-ITI single-trial spectrogram for a single trial. An increase in power can be seen during the response phase compared to the fixation phase.

ship to behavior. However, the full range of the gamma band (up to $200 \mathrm{~Hz}$ ) has been associated with motor control, as seen in previous literature..$^{53,55-59}$

Specifically in the amygdala, there is limited research exploring the role of the gamma band, particularly in humans. In a 2012 study of 6 patients undergoing presurgical evaluation, Sato et al. reported amygdala gamma-band power modulation in response to human faces compared with nonfacial stimuli, indicating that the gamma band may be involved in facial processing. ${ }^{46}$ Low gamma (25$35 \mathrm{~Hz}$ ) oscillations have also been shown to be involved in olfactory processing in humans ${ }^{43}$ In that study, patients had an odor-induced amygdala gamma-band modulation that lasted for the full duration of inspiration. Given these heterogeneous findings, it is clear that this is an area that warrants further study to elucidate the variety of roles that the gamma band may play in subcortical structures.

In this study, we also observed statistically significant modulation in the beta, theta, and alpha bands in a small number of patients. Throughout the motor system, the beta band is known to play a role in movement execution and inhibition. ${ }^{67-70}$ It is possible that the beta-band drop we observed during the response phase is related to its established motor function in other parts of the brain, but we are unable to draw any conclusions given that we observed this effect in only 3 patients. The role of the theta and alpha bands are less studied in movement execution, and we only observed this modulation in a single patient. Studying these frequency ranges in the amygdala in a larger number of participants is an important area of future study.

Here, we briefly explore some of the limitations of our study. Of note, the effect that we describe was seen in 5 of 8 patients. Additionally, as seen in Table 2, the contiguous frequency ranges that were implicated in the execution of voluntary movement varied across patients. While the group analysis points out common power characteristics among patients, a limitation of this analysis when combining and averaging trials from all patients is that the overall 
effect can be weighted toward patients with more successful trials, and toward patients with wider frequency ranges in their individual response. In this study, the number and placement of electrodes were individualized for each patient based on their suspected seizure origin in a manner consistent with surgical planning. It is possible that the electrode contacts of interest may be located in different subregions of the amygdala across the various patients. However, we believe that the grouping of trials across patients provides a useful illustration of the average observed modulation even in the context of this limitation. It is also known that the amygdalae of patients with epilepsy differ from those of healthy controls, ${ }^{71}$ so it is an open question as to whether these findings would also be seen in patients without epilepsy. Given that every patient conducted the trial with their right hand, the effect that handedness has on these results is unclear. It is possible that some of the variation we see in frequency ranges or magnitude in modulation in the gamma band may be explained by not taking handedness into account. Although the center-out direct-reach task is designed to isolate the effect of arm movement, it is possible that other actions that we did not track may have influenced the results. There is evidence that gamma oscillations may be involved in eye movement ${ }^{72}$ although these findings were in the occipital region of the brain rather than the amygdala. However, as mentioned previously, the amygdala may play a role in visual processing, although those findings were limited to human faces. Lastly, there was no ability to quantify the magnitude of movement with the amount of power modulation observed. Future studies may examine this with the use of accelerometers or other tools to quantify movement and correlate it with the magnitude of power change.

\section{Conclusions}

Our work indicates that the gamma band plays a significant role in the execution of voluntary movement in the human amygdala and may be a suitable neural signal for use in a motor BCI system. It is possible that motor tasks, in addition to the classic center-out direct-reach task, may be warranted to gain a better understanding of how the amygdala is involved in movement beyond just movement execution. Potential tasks may include motor inhibition and directional motor tasks, which would help to characterize the amygdala's function in various motor behaviors. Although previous work has indicated that the amygdala is connected to various parts of the motor system, there is work to be done to understand temporal and functional interactions between these various areas. Further study will be key in developing a motor BCI system. We believe that direct intracranial electrophysiology may be useful in studying these interactions.

\section{Acknowledgments}

We wish to acknowledge the generous support of the National Center for Advancing Translational Science (NCATS) of the U.S. National Institutes of Health (KL2TR001854), the Tianqiao and Chrissy Chen Brain-Machine Interface Center at Caltech, the Meira and Shaul G. Massry Foundation, and the Taiwan-USC Postdoctoral Fellowship Program.

\section{References}

1. Andersen RA, Burdick JW, Musallam S, et al. Cognitive neural prosthetics. Trends Cogn Sci. 2004;8(11):486-493.

2. Apuzzo ML, Liker MA, Amar AP. Honored guest presentation: neurorestoration and the emergence of molecular and cellular neurosurgery. Clin Neurosurg. 2002;49:274-315.

3. Elder JB, Hoh DJ, Oh BC, Heller AC, Liu CY, Apuzzo ML. The future of cerebral surgery: a kaleidoscope of opportunities. Neurosurgery. 2008;62(suppl_3):SHC1555-SHC1582.

4. Lee B, Liu CY, Apuzzo ML. A primer on brain-machine interfaces, concepts, and technology: a key element in the future of functional neurorestoration. World Neurosurg. 2013;79(3-4):457-471.

5. Salanova V, Morrell MJ. Responsive brain stimulation for treatment of medically intractable mesial temporal epilepsy in adults: results of a subset analysis from the RNS ${ }^{\mathrm{TM}}$ System pivotal investigation in epilepsy. Paper presented at: 62nd AAN Annual Meeting; April 10-17, 2010; Toronto, ON, Canada.

6. Hariz M. Twenty-five years of deep brain stimulation: celebrations and apprehensions. Mov Disord. 2012;27(7):930933.

7. Duncan PW, Goldstein LB, Horner RD, et al. Similar motor recovery of upper and lower extremities after stroke. Stroke. 1994;25(6):1181-1188.

8. Goldstein LB, Davis JN. Restorative neurology. Drugs and recovery following stroke. Stroke. 1990;21(11):1636-1640.

9. Toscano J. Prevention of neurological deterioration before admission to a spinal cord injury unit. Paraplegia. 1988;26(3):143-150.

10. Georgopoulos AP, Schwartz AB, Kettner RE. Neuronal population coding of movement direction. Science. 1986;233(4771):1416-1419.

11. Nicolelis MA. Brain-machine interfaces to restore motor function and probe neural circuits. Nat Rev Neurosci. 2003;4(5):417-422.

12. Schwartz AB, Kettner RE, Georgopoulos AP. Primate motor cortex and free arm movements to visual targets in threedimensional space. I. Relations between single cell discharge and direction of movement. J Neurosci. 1988;8(8):2913-2927.

13. Scott SH. Inconvenient truths about neural processing in primary motor cortex. J Physiol. 2008;586(5):1217-1224.

14. Hwang EJ, Andersen RA. Brain control of movement execution onset using local field potentials in posterior parietal cortex. J Neurosci. 2009;29(45):14363-14370.

15. Kakei S, Hoffman DS, Strick PL. Muscle and movement representations in the primary motor cortex. Science. 1999;285(5436):2136-2139.

16. Mulliken GH, Musallam S, Andersen RA. Decoding trajectories from posterior parietal cortex ensembles. J Neurosci. 2008;28(48):12913-12926.

17. Musallam S, Corneil BD, Greger B, et al. Cognitive control signals for neural prosthetics. Science. 2004;305(5681):258262.

18. Snyder LH, Batista AP, Andersen RA. Coding of intention in the posterior parietal cortex. Nature. 1997;386(6621):167-170.

19. Sakata H, Taira M, Murata A, Mine S. Neural mechanisms of visual guidance of hand action in the parietal cortex of the monkey. Cereb Cortex. 1995;5(5):429-438.

20. Andersen RA, Buneo CA. Intentional maps in posterior parietal cortex. Annu Rev Neurosci. 2002;25(1):189-220.

21. Gail A, Andersen RA. Neural dynamics in monkey parietal reach region reflect context-specific sensorimotor transformations. J Neurosci. 2006;26(37):9376-9384.

22. Mountcastle VB, Lynch JC, Georgopoulos A, et al. Posterior parietal association cortex of the monkey: command functions for operations within extrapersonal space. J Neurophysiol. 1975;38(4):871-908.

23. Chang SW, Dickinson AR, Snyder LH. Limb-specific repre- 
sentation for reaching in the posterior parietal cortex. $J \mathrm{Neu}$ rosci. 2008;28(24):6128-6140.

24. Chang SW, Snyder LH. The representations of reach endpoints in posterior parietal cortex depend on which hand does the reaching. J Neurophysiol. 2012;107(9):2352-2365.

25. Mulliken GH, Musallam S, Andersen RA. Forward estimation of movement state in posterior parietal cortex. Proc Natl Acad Sci U S A. 2008;105(24):8170-8177.

26. LaBar KS, Gatenby JC, Gore JC, et al. Human amygdala activation during conditioned fear acquisition and extinction: a mixed-trial fMRI study. Neuron. 1998;20(5):937-945.

27. Adolphs R, Tranel D, Damasio H, Damasio A. Impaired recognition of emotion in facial expressions following bilateral damage to the human amygdala. Nature. 1994;372(6507):669-672.

28. Anderson AK, Phelps EA. Lesions of the human amygdala impair enhanced perception of emotionally salient events. Nature. 2001;411(6835):305-309.

29. Toschi N, Duggento A, Passamonti L. Functional connectivity in amygdalar-sensory/(pre)motor networks at rest: new evidence from the Human Connectome Project. Eur J Neurosci. 2017;45(9):1224-1229.

30. Grèzes J, Valabrègue R, Gholipour $\mathrm{B}$, Chevallier $\mathrm{C}$. A direct amygdala-motor pathway for emotional displays to influence action: a diffusion tensor imaging study. Hum Brain Mapp. 2014;35(12):5974-5983.

31. Péron J, Frühholz S, Ceravolo L, Grandjean D. Structural and functional connectivity of the subthalamic nucleus during vocal emotion decoding. Soc Cogn Affect Neurosci. 2016;11(2):349-356.

32. Yang J, Yin Y, Svob C, et al. Amygdala atrophy and its functional disconnection with the cortico-striatal-pallidal-thalamic circuit in major depressive disorder in females. PLoS One. 2017;12(1):e0168239.

33. Gilat M, Ehgoetz Martens KA, Miranda-Domínguez O, et al. Dysfunctional limbic circuitry underlying freezing of gait in Parkinson's disease. Neuroscience. 2018;374:119-132.

34. Youngerman BE, Khan FA, McKhann GM. Stereoelectroencephalography in epilepsy, cognitive neurophysiology, and psychiatric disease: safety, efficacy, and place in therapy. Neuropsychiatr Dis Treat. 2019;15:1701-1716.

35. Li G, Jiang S, Paraskevopoulou SE, et al. Optimal referencing for stereo-electroencephalographic (SEEG) recordings. Neuroimage. 2018;183:327-335.

36. Buzsáki G, Draguhn A. Neuronal oscillations in cortical networks. Science. 2004;304(5679):1926-1929.

37. Buzsaki G. Rhythms of the Brain. Oxford University Press; 2006.

38. Cantero JL, Atienza M. The role of neural synchronization in the emergence of cognition across the wake-sleep cycle. Rev Neurosci. 2005;16(1):69-83.

39. Nunez PL. Toward a quantitative description of large-scale neocortical dynamic function and EEG. Behav Brain Sci. 2000;23(3):371-437.

40. Knyazev GG. Motivation, emotion, and their inhibitory control mirrored in brain oscillations. Neurosci Biobehav Rev. 2007;31(3):377-395.

41. Zumsteg D, Wieser HG. Presurgical evaluation: current role of invasive EEG. Epilepsia. 2000;41(suppl 3):S55-S60.

42. Oya H, Kawasaki H, Howard MA III, Adolphs R. Electrophysiological responses in the human amygdala discriminate emotion categories of complex visual stimuli. J Neurosci. 2002;22(21):9502-9512.

43. Jung J, Hudry J, Ryvlin P, et al. Functional significance of olfactory-induced oscillations in the human amygdala. Cereb Cortex. 2006;16(1):1-8.

44. Rutishauser U, Mamelak AN, Adolphs R. The primate amygdala in social perception-insights from electrophysiological recordings and stimulation. Trends Neurosci. 2015;38(5):295306.
45. Karalis N, Dejean C, Chaudun F, et al. 4-Hz oscillations synchronize prefrontal-amygdala circuits during fear behavior. Nat Neurosci. 2016;19(4):605-612.

46. Sato W, Kochiyama T, Uono S, et al. Temporal profile of amygdala g oscillations in response to faces. J Cogn Neurosci. 2012;24(6):1420-1433.

47. Samson RD, Lester AW, Duarte L, et al. Emergence of b-band oscillations in the aged rat amygdala during discrimination learning and decision making tasks. eNeuro. 2017:4(5):ENEURO.0245-17.2017.

48. Dalal SS, Hamamé CM, Eichenlaub JB, Jerbi K. Intrinsic coupling between gamma oscillations, neuronal discharges, and slow cortical oscillations during human slow-wave sleep. J Neurosci. 2010;30(43):14285-14287.

49. Bojak I. Gamma rhythm, neural population models of the. In: Jaeger D, Jung R, eds. Encyclopedia of Computational Neuroscience. Springer; 2014.

50. Fitzgerald PJ, Watson BO. Gamma oscillations as a biomarker for major depression: an emerging topic. Transl Psychiatry. 2018;8(1):177.

51. Miller KJ, Honey CJ, Hermes D, et al. Broadband changes in the cortical surface potential track activation of functionally diverse neuronal populations. Neuroimage. 2014;85(pt 2):711-720.

52. Nir Y, Mukamel R, Dinstein I, et al. Interhemispheric correlations of slow spontaneous neuronal fluctuations revealed in human sensory cortex. Nat Neurosci. 2008;11(9):1100-1108.

53. Ray S, Crone NE, Niebur E, et al. Neural correlates of highgamma oscillations $(60-200 \mathrm{~Hz})$ in macaque local field potentials and their potential implications in electrocorticography. J Neurosci. 2008;28(45):11526-11536.

54. Miller KJ, Sorensen LB, Ojemann JG, den Nijs M. Power-law scaling in the brain surface electric potential. PLoS Comput Biol. 2009;5(12):e1000609.

55. Crone NE, Miglioretti DL, Gordon B, Lesser RP. Functional mapping of human sensorimotor cortex with electrocorticographic spectral analysis. II. Event-related synchronization in the gamma band. Brain. 1998;121(pt 12):2301-2315.

56. Miller KJ, Leuthardt EC, Schalk G, et al. Spectral changes in cortical surface potentials during motor movement. J Neurosci. 2007;27(9):2424-2432.

57. Joundi RA, Jenkinson N, Brittain J-S, et al. Driving oscillatory activity in the human cortex enhances motor performance. Curr Biol. 2012;22(5):403-407.

58. Engelhard B, Ozeri N, Israel Z, et al. Inducing g oscillations and precise spike synchrony by operant conditioning via brain-machine interface. Neuron. 2013;77(2):361-375.

59. Pfurtscheller G, Graimann B, Huggins JE, et al. Spatiotemporal patterns of beta desynchronization and gamma synchronization in corticographic data during self-paced movement. Clin Neurophysiol. 2003;114(7):1226-1236.

60. Jenkinson N, Kühn AA, Brown P. g oscillations in the human basal ganglia. Exp Neurol. 2013;245:72-76.

61. Brainard DH. The psychophysics toolbox. Spat Vis. 1997;10(4):433-436.

62. Pelli DG. The VideoToolbox software for visual psychophysics: transforming numbers into movies. Spat Vis. 1997;10(4):437-442.

63. Kleiner M, Brainard D, Pelli D. What's new in Psychtoolbox-3? Psychology. 2007;36(14):1-16.

64. Bokil H, Andrews P, Kulkarni JE, et al. Chronux: a platform for analyzing neural signals. J Neurosci Methods. 2010;192(1):146-151.

65. Haukoos JS, Lewis RJ. Advanced statistics: bootstrapping confidence intervals for statistics with "difficult" distributions. Acad Emerg Med. 2005;12(4):360-365.

66. Voon V, Brezing C, Gallea C, Hallett M. Aberrant supplementary motor complex and limbic activity during motor preparation in motor conversion disorder. Mov Disord. 2011;26(13):2396-2403. 
67. Salmelin R, Hari R. Spatiotemporal characteristics of sensorimotor neuromagnetic rhythms related to thumb movement Neuroscience. 1994;60(2):537-550.

68. Brown P. Abnormal oscillatory synchronisation in the motor system leads to impaired movement. Curr Opin Neurobiol. 2007;17(6):656-664.

69. Engel AK, Fries P. Beta-band oscillations-signalling the status quo? Curr Opin Neurobiol. 2010;20(2):156-165.

70. Jenkinson N, Brown P. New insights into the relationship between dopamine, beta oscillations and motor function. Trends Neurosci. 2011;34(12):611-618.

71. Cendes F, Andermann F, Gloor P, et al. MRI volumetric measurement of amygdala and hippocampus in temporal lobe epilepsy. Neurology. 1993;43(4):719-725.

72. Nagasawa T, Matsuzaki N, Juhász C, et al. Occipital gammaoscillations modulated during eye movement tasks: simultaneous eye tracking and electrocorticography recording in epileptic patients. Neuroimage. 2011;58(4):1101-1109.

\section{Disclosures}

The authors report no conflict of interest concerning the materials or methods used in this study or the findings specified in this paper.

\section{Author Contributions}

Conception and design: Gogia, Martin del Campo-Vera, Chen, Sebastian, Nune, Kramer, Liu, Kellis, B Lee. Acquisition of data: Gogia, Martin del Campo-Vera, Chen, Sebastian, Nune, Kramer, MB Lee, Tafreshi, Barbaro, Kellis, B Lee. Analysis and interpretation of data: Gogia, Martin del Campo-Vera, Chen, Sebastian, Nune, Kramer, MB Lee, Tafreshi, Barbaro, Kellis, B Lee. Drafting the article: Gogia, Martin del Campo-Vera, Chen, Sebastian, Kramer, MB Lee, Tafreshi, Barbaro, Kellis, B Lee. Critically revising the article: all authors. Reviewed submitted version of manuscript: Gogia, Martin del Campo-Vera, Chen, Sebastian, MB Lee, Tafreshi, Barbaro, Liu, Kellis, B Lee. Approved the final version of the manuscript on behalf of all authors: Gogia. Statistical analysis: Gogia, Martin del CampoVera, Chen, Sebastian, Kellis, B Lee. Administrative/technical/ material support: Nune, Liu, Kellis, B Lee. Study supervision: Nune, Kramer, Liu, Kellis, B Lee.

\section{Correspondence}

Angad S. Gogia: Keck School of Medicine, University of Southern California, Los Angeles, CA. angadgog@usc.edu. 